\title{
Dramatic Response to Lidocaine Infusion for Pain from Brachial Plexus Avulsion InJury
}

\author{
Philippa Hawley, FRCPC ${ }^{1,2}$, Julia Ridley, MD ${ }^{1,2}$, and Marc Youngson ${ }^{1}$
}

Background: Traumatic brachial plexus avulsion injury (tBPI) can cause a severe chronic pain syndrome that is very difficult to treat. Though lidocaine has been shown to be effective for other pain syndromes, effectiveness in tBPI has not previously been reported.

Case Report: A 55-year-old man with tBPI had severe pain and minimal relief with numerous analgesic agents. He was able to access intravenous lidocaine as he was being treated at a cancer center, and had a sustained response to $7 \mathrm{mg} / \mathrm{kg}$ given over an hour.

Conclusion: There is potential for a single bolus intravenous lidocaine infusion to provide good pain control sustained over many months in patients with traumatic brachial plexus injury. An adequate serum concentration of lidocaine is required for analgesic effect. If initial doses of lidocaine are tolerated but ineffective, higher doses may still be beneficial.

Key words: Brachial plexus avulsion, lidocaine, lignocaine, pain management, palliative care, therapeutics, trauma

\section{BACKGROUND}

Traumatic brachial plexus injury (tBPI) can lead to severe chronic neuropathic pain which may be resistant to standard treatments and lead to great suffering. Lidocaine infusion has been reported to be of benefit in a variety of pain syndromes, but has not yet been reported for tBPI.

\section{CASE}

A 55-year-old healthy right-handed man was struck by a car while walking, thrown into the windshield and then onto the ground. He was pulling a wheeled suitcase at the time of impact. His right arm was pulled backwards. There was no loss of consciousness. He sustained lacerations, an undisplaced right tibial plateau fracture, an avulsion fracture of his right ankle, and a mild compression fracture of the T7 vertebral body. CT head was normal. There were no fractures of the bones of the arm or shoulder, and no significant soft tissue injury to the arm itself below the shoulder/axilla.

Following initial management, he had persistent severe pain in the right elbow. Six weeks after the injury, he had tingling and numbness in the fourth and fifth fingers of his right hand, medial aspect of his forearm, and elbow. Movement of his arm was severely limited by pain, but there was no motor loss. The pain was clearly neuropathic.

Three months after the injury, nerve conduction studies and electromyogram (EMG) showed acute denervation of multiple muscle groups and injury to the ulnar, radial, and median nerves, consistent with a brachial plexus traction injury. There was widespread right arm weakness, sensory loss, and absent arm reflexes. Pain extended from his right scapula through to his medial forearm and palm, with tingling and burning of both palmar and dorsal aspects of his hand, including all

From: 'BC Cancer Pain \& Symptom Management/Palliative Care Program, Vancouver, Canada; ${ }^{2}$ University of British Columbia Division of Palliative Care, Vancouver, Canada

Corresponding Author: Philippa Hawley, FRCPC, E-mail: phawley@bccancer.bc.ca Disclaimer: There was no external funding in the preparation of this manuscript.

Conflict of interest: Each author certifies that he or she, or a member of his or her immediate family, has no commercial association (i.e., consultancies, stock ownership, equity interest, patent/licensing arrangements, etc.) that might pose a conflict of interest in connection with the submitted manuscript. Accepted: 2021-03-31, Published: 2021-06-14 
fingers and thumb. Imaging showed swelling of C6, 7, 8 , and T1 nerve roots.

He was started on gabapentin, which was increased with partial benefit over 3 months to $800 \mathrm{mg} 3$ times a day. Dosing was limited by sedation. Topical capsaicin, oral amitriptyline, oral medical cannabis extracts, and a topical cannabis-based cream were all tried but ineffective. He was reluctant to use opioids.

Eight months after the injury he was admitted to the hospital with weight loss, nausea, vomiting, and diarrhea, and was found to have a large abdominal mass. He was diagnosed with an aggressive large B-cell lymphoma with retroperitoneal lymphadenopathy and pelvic bone metastases. The lymphoma was treated with chemotherapy (CHOP-R), with vincristine omitted due to recent nerve injury. He developed cranial nerve V1 shingles managed promptly with valacyclovir. Gabapentin was switched to pregabalin $150 \mathrm{mg} 3$ times a day. His severe arm pain persisted throughout, despite high-dose steroids with his chemotherapy protocol.

Ten months after the injury he was referred to the BC Cancer Vancouver Centre's Pain \& Symptom Management/Palliative Care (PSMPC) clinic with persistent severe pain and loss of function of his right arm. He was started on duloxetine $30 \mathrm{mg}$ at bedtime, increased to $60 \mathrm{mg}$ which was well-tolerated and felt to be helpful. Oxycodone $5 \mathrm{mg}$ and a compounded topical amitriptyline, ketoprofen, and ketamine cream were ineffective. Other opioids and higher doses were not tried, due to patient preference.

He was referred to a non-cancer pain clinic, where he was believed not to have complex regional pain syndrome.

A month later he was reviewed at the PSMPC clinic and reported mild improvement in his pain with duloxetine, from 7 to 10 of 10 down to 5 to 6 of 10 , but was still in significant pain. He agreed to try low-dose methadone, $1 \mathrm{mg}$ every 8 hours, which was somewhat helpful. Over the next 9 months, however, the pain worsened and the dose of methadone was slowly increased to his maximum tolerated dose of $7.5 \mathrm{mg}$ every 8 hours, limited by drowsiness, still with limited effectiveness.

Unfortunately, his lymphoma progressed rapidly, and he entered an oncology clinical trial. At this point, a year following his injury, his pain ranged from 3 to 8 of 10 . He continued to receive physiotherapy and was compliant with recommended exercises. There was no improvement in sensory nerve latencies, but his motor function improved slightly.
His pain subsequently escalated, markedly impairing his quality of life. Increased doses of methadone were not tolerated. Hydromorphone and multiple courses of corticosteroids as part of his cancer treatment were ineffective for pain, which he now reported at 8 to 10 of 10, with frequent lancinating pain "spasms." Interventional procedures were contemplated but in view of his relatively immunocompromised state it was felt preferable to avoid invasive procedures and particularly any risk from foreign body insertion, such as an epidural catheter. Our center also does not have on-site anesthesia support other than for our surgical suite and for administration of radiotherapy to children.

Treatment with lidocaine $5 \mathrm{mg} / \mathrm{kg}$ intravenously over one hour produced no analgesic benefit, but no lidocaine-specific side effects (i.e., metallic taste, perioral numbness or tingling) either. Duloxetine was switched to nortriptyline and escalated to $60 \mathrm{mg}$ at bedtime over a month. Review by neurology noted new left median nerve compression in the carpal tunnel, managed with a wrist splint, and new right ulnar nerve compression at the elbow, which was mild and managed conservatively.

Myokymia of the arm muscles was now quite prominent. He also displayed some apraxia and stereognosis. Magnetic resonance imaging of the cervical spine and right brachial plexus showed no evidence of lymphoma involvement at any time.

Five weeks following his initial lidocaine infusion (20 months after the injury) it was decided to try lidocaine again, at a dose of $7 \mathrm{mg} / \mathrm{kg}$ over one hour. This infusion produced a dramatic benefit, with pain in his forearm, upper arm, and shoulder completely resolving (0 of 10) by completion of the infusion, and pain left in just his fingers reducing initially to a maximum of 5 of 10 , then further declining to 2-3 of 10 over the next 4 months. Despite an expectation that the effect of the lidocaine would be temporary, the disabling arm pain did not recur.

His hand pain persisted but was mild and manageable with methadone $7 \mathrm{mg}$ every 8 hours and pregabalin $150 \mathrm{mg}$ every 8 hours. The nortriptyline was tapered by $10 \mathrm{mg}$ weekly until discontinued, with no loss of pain control. The patient died comfortably at home from lymphoma approximately 7 months after the second lidocaine infusion, over 2 years following the tBPI.

This patient had good supports, no adjustment disorder, depression, or anxiety disproportionate to his circumstances. He had no spiritual or existential concerns and maintained his excellent sense of humor. It was not 
felt that there were any reversible nonphysical contributors to his pain, which was consistent in reported location and quality. He had no difficulty using a 0-10 scale or describing his pain qualitatively.

\section{DISCUSSION}

Any treatment can produce a placebo effect, especially one requiring a procedure, but this is highly unlikely in this case, as the initial $5 \mathrm{mg} / \mathrm{kg}$ infusion had no effect. "Total pain" can show significant placebo responses where reassurance and support are part of a medical intervention, but there were no psychological or social issues of concern for this patient, and no changes in circumstance or events that occurred around the time of pain response. Nerve injuries can also resolve over time by themselves, but it is highly unlikely that this patient's relief was due to nerve recovery alone, as it was dramatic and immediate at the time of expected peak blood level. Any effect of the serotonin-norepinephrine reuptake inhibitor or tricyclic would have been expected to occur much sooner, and no other medical interventions coincided with the response.

tBPIs can be treated surgically using nerve and musculoskeletal reconstruction procedures, if diagnosed and treated within a 6 -month timeframe $(1,2)$. Neuropathic pain has long been recognized as a significant problem following tBPI (3), occurring in $50 \%$ to $80 \%$ of patients $(4,5)$. A literature review yields little specific information of the best modalities for treatment. Medications commonly used for neuropathic pain are suggested, but effectiveness is poor in $\operatorname{tBPI}(2,6)$. There are reports of relief with dorsal root entry zone (DREZ) lesions (7) and spinal cord stimulation $(4,8)$. We were unable to find any publications referencing systemic lidocaine for pain related specifically to $\mathrm{tBPI}$.

It is possible that despite the absence of vasomotor abnormalities or skin changes, this patient developed a component of complex regional pain syndrome (CRPS), and there is some evidence for benefit from lidocaine infusion in CRPS (9). A study in 16 patients with CRPS compared computer-controlled short intravenous lidocaine infusions (20 minutes at different target blood levels) with diphenhydramine, and found benefit for lidocaine, particularly a decrease in cold-induced pain, allodynia, and spontaneous pain. The remainder of the medical literature on lidocaine infusions is for a variety of other pain syndromes $(10,11)$.

Intravenous lidocaine has been shown to block sodium channels, uncouple G protein, block NMDA re- ceptors, reduce circulating inflammatory cytokines, and prevent or reduce secondary hyperalgesia and central sensitization (11). The dramatic benefit of achieving a therapeutic blood level in this patient illustrates the need to titrate lidocaine infusions individually. Therapeutic level ranges have been suggested, but as levels are not actually able to be measured in real time during an infusion, and the same dose can lead to different blood levels in different people, the concept of therapeutic level is a clinical one rather than an actual number that can be targeted in any one infusion (12). The dose should be increased incrementally until either analgesia occurs (successful trial), or lidocaine-specific side effects occur, indicating that a potentially therapeutic blood level has been achieved; in the absence of analgesia, the trial can be deemed failed (13). Close clinical monitoring for mild lidocaine-specific side effects is a safe way of determining if a potentially therapeutic blood level has been achieved. Lack of response to an infusion that produces no such side effects should not be taken to mean that the individual's pain is lidocaineinsensitive, as a therapeutic blood level may not have been reached.

This case demonstrates how the cycle of secondary pain sensitization and "wind-up" following nerve injury can be broken, with sustained pain relief lasting well beyond any presence of the drug itself $(10,14)$. Despite a recent systematic review (14) concluding that there was no evidence from randomized controlled trials of lidocaine infusion having a prolonged duration of effect, this phenomenon has been observed frequently in clinical practice (14), one study reporting pain relief lasting at least 3 weeks in $8 \%$ of 98 tertiary chronic pain clinic patients.

Access to lidocaine infusions is very restricted, at least in Canada, with widespread unfounded concern about risk of cardiac arrhythmias and other serious toxicities being a barrier to access. This is despite the side effects of lidocaine being predictable and easily identified, safely managed by dose adjustment. Electrocardiographic (ECG) monitoring is not required in this context. This patient was only offered lidocaine infusion through having cancer, despite review in neurology and chronic non-cancer pain settings multiple times prior to his cancer diagnosis, with clear documentation of escalating pain and failed trials of non-opioid oral neuropathic pain adjuvant medications.

A preference to avoid opioids is not unusual in the context of public knowledge of potential harms of 
opioids. It is possible that a trial of methadone earlier following the nerve injury may have reduced secondary sensitization; however, the eventual response to methadone was only partial.

Modern oncology treatments are leading to prolonged survivorship, with a high prevalence of chronic pain syndromes, especially neuropathic postsurgical pain, radiculopathy from spinal disease, and peripheral neuropathy caused by cancer treatments (15). These pains are often relatively opioid-resistant, and prolonged opioid therapy can lead to tolerance and opioidinduced hyperalgesia. The small but not insignificant risk of concurrent or iatrogenic opioid use disorder is being recognized (16). Non-opioid interventions (both pharmacological and nonpharmacological) are increasingly required for management of cancer pain. The skills and services generally found in non-cancer pain clinic settings are increasingly relevant to palliative care services, particularly outpatient clinics, where patients may have prolonged interaction with both palliative care and disease management teams.

Though sadly this patient's cancer ended his life, his journey from the nerve injury through to the diagnosis

\section{REFERENCES}

1. Franzblau LE, Maynard M, Chung KC, Yang LJ. Medical treatment decision making after total avulsion brachial plexus injury: A qualitative study. J Neurosurg 2015; 122:1413-1420.

2. Giuffre JL, Kakar S, Bishop AT, Spinner RJ, Shin AY. Current concepts of the treatment of adult brachial plexus injuries [published correction appears in J Hand Surg Am 2010; 35:1226. Kakar, Sanjiv (corrected to Kakar, Sanjeev)]. J Hand Surg Am 2010; 35:678688.

3. Bruxelle J, Travers V, Thiebaut JB. Occurrence and treatment of pain after brachial plexus injury. Clin Orthop Relat Res 1988; 237:87-95.

4. Brill S, Aryeh IG. Neuromodulation in the management of pain from brachial plexus injury. Pain Physician 2008; 11:81-85.

5. Ciaramitaro P, Padua L, Devigili G, et al. Prevalence of neuropathic pain in patients with traumatic brachial plexus injury: A multicenter prospective hospital-based study. Pain Med 2017; 18:24282432.

6. Davis G, Curtin CM. Management of pain in complex nerve injuries. Hand Clin 2016; 32:257-262.

7. Samii M, Bear-Henney S, Lüdemann W, Tatagiba M, Blömer $U$. Treatment of refractory pain after brachial plexus avulsion with dorsal root entry zone lesions. Neurosurgery 2001; 48:1269-1277.

8. Floridia D, Cerra F, Guzzo G, et al. Treatment of pain post-brachial plexus injury using high-frequency spinal cord stimulation. J Pain Res 2018; 11:2997-3002.

9. Wallace MS, Ridgeway BM, Leung AY, Gerayli A, Yaksh TL. Concentration-effect relationship of intravenous lidocaine on the allo- and management of his lymphoma illustrates the benefits of a prognosis-independent integrated supportive cancer care program including rehabilitation services (17), known as the "bow tie" model. The possibility of cure was not an unreasonable expectation for a large part of his journey. A good outcome from management of his injury-related pain created trust and a facilitated a collaborative relationship between the patient and his family, the PSMPC team, oncology team, and ultimately his home hospice team.

\section{CONCLUSION}

This case illustrates a number of key learning points.

- Brachial plexus avulsion pain can be very resistant to conventional analgesics, and intravenous lidocaine may offer relief.

- For a lidocaine infusion to provide analgesia, an individually clinically determined threshold blood level needs to be reached.

- Lidocaine infusion can dramatically reverse hyperalgesia and secondary sensitization, and should be more widely available.

dynia of complex regional pain syndrome types I and II. Anesthesiology 2000; 92:75-83.

10. Zhu B, Zhou X, Zhou Q, Wang H, Wang S, Luo K. Intra-venous lidocaine to relieve neuropathic pain: A systematic review and meta-analysis. Front Neurol 2019; 10:954

11. Kandil E, Melikman E, Adinoff B. Lidocaine infusion: A promising therapeutic approach for chronic pain. J Anesth Clin Res 2017; 8:697.

12. Eipe N, Gupta S, Penning J. Intravenous lidocaine for acute pain: An evidence-based clinical update. BJA Educ 2016; 16:292-298.

13. Peixoto RD, Hawley P. Intravenous lidocaine for cancer pain without electrocardiographic monitoring: A retrospective review. J Palliat Med 2015; 18:373-377.

14. Attal N, Rouaud J, Brasseur L, Chauvin M, Bouhassira D. Systemic lidocaine in pain due to peripheral nerve injury and predictors of response. Neurology 2004; 62:218-225.

15. lacob E, Hagn EE, Sindt J. Tertiary care clinical experience with intravenous lidocaine infusions for the treatment of chronic pain. Pain Med 2018; 19:1245-1253.

16. Jiang $\mathrm{C}$, Wang $\mathrm{H}$, Wang $\mathrm{Q}$, et al. Prevalence of chronic pain and high-impact chronic pain in cancer survivors in the United States. JAMA Oncol 2019; 5:1224-1226.

17. Dalal S, Bruera E. Pain management for patients with advanced cancer in the opioid epidemic era. Am Soc Clin Oncol Educ Book 2019; 39:24-35

18. Hawley P. The bow tie model of 21 st century palliative care. J Pain Symptom Manage 2014; 47:e2-e5. 